\title{
Radiologic features of pulmonary cement embolism after percutaneous vertebroplasty in a case report
}

\author{
๑5atih Ateş, ๑Abidin Kılınçer, ๑Hakan Cebeci, $₫$ Mehmet Sedat Durmaz \\ Selçuk University, School of Medicine, Department of Radiology, Konya, Turkey
}

Cite this article as: Ateş F, Kılınçer A, Cebeci H, Durmaz MS. Radiologic features of pulmonary cement embolism after percutaneous vertebroplasty in a case report. Anatolian Curr Med J 2021; 3(1): 81-84.

\begin{abstract}
Percutaneous vertebroplasty is a well-known treatment modality for vertebral compression fractures caused by osteoporosis and malignant metastases. The procedure involves the injection of poly methyl methacrylate (PMMA, bone cement), in to the desired vertebral body. Pulmonary cement embolism (PCE) refers to the embolization of PMMA into the pulmonary arteries. Patients are often asymptomatic and it may be detected incidentally for imaging performed for another reason. The most common symptoms of pulmonary cement embolization are chest pain and breathlessness, and rarely it can present as acute respiratory distress syndrome. In this case, we aimed to present a 73-year-old male patient of PCE with computed tomography (CT) images. We want to raise the awareness that all patients undergoing vertebroplasty procedure should have routine chest $\mathrm{X}$-ray after the procedure. However, $\mathrm{CT}$ is the best modality to determine PCE.
\end{abstract}

Keywords: Pulmonary cement embolism, vertebroplasty, CT, poly methyl methacrylate

\section{INTRODUCTION}

Vertebroplasty is the procedure of percutaneous implantation of poly methyl methacrylate (PMMA) into the vertebral corpus with radiological imaging in situations such as compression fracture or height loss in the vertebra after trauma or many diseases (osteoporosis, metastatic lesions, etc.) (1). The procedure is defined as injection of PMMA (bone cement) into the desired vertebral body. PMMA is often used in vertebroplasty and is structurally an acrylic cement $(1,2)$. Pulmonary cement embolism (PCE) describes the embolization of PMMA to the lungs. After being injected in to the vertebral body, PMMA cement can reach the pulmonary artery via the paravertebral venous system and the azygos vein, causing PCE (3). Although patients usually do not describe symptomas and are detected incidentally on imaging performed for another reason, there are few reports of serious and fatal outcomes in the literature $(4,5)$. In this case report, we aimed to present PCE in a patient diagnosed with computed tomography $(\mathrm{CT})$ in the light of the literature.

\section{CASE}

A 73-year-old male patient was admitted to the clinic by dyspnea complaints. The patient also had chronic obstructive pulmonary disease (COPD), hypertension, and diabetes mellitus (DM) for 10 years. Vertebroplasty was performed when a compression fracture in the L2 vertebra corpus was detected in the patient who had a history of falling from a wooden ladder 3 months ago, severe pain in his waist, and severe tenderness with palpation in the thoracolumbar region. The patient, who had frequent COPD attacks, was admitted to the emergency department with dyspnea complaints. Direct radiography and thorax CT was applied and laboratory examinations were analyzed. Vertebroplasty was detected in the L2 vertebra corpus by CT (Figure 1-2). PCE was observed in the left paravertebral venous structures. Also, hyperdense appearances compatible with cement embolism matching the vascular trace were observed in the upper and middle lobes of the right lung and to a lesser extent in the anterior segment of the left lung upper lobe (Figure 2-3).Our patient was treated as PCE with anticoagulation but it was determined that the symptoms of the patient were secondary to the COPD attack. 


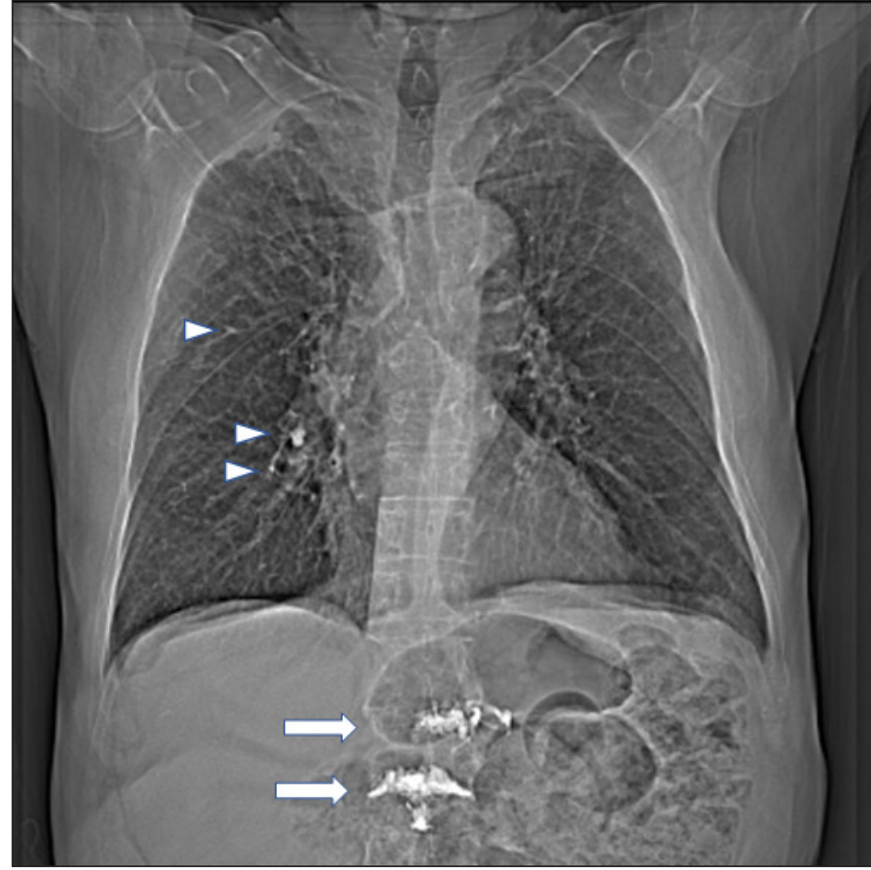

Figure 1. The L2 vertebroplasty was shown by white arrows in the topographic image. Pulmonary cement embolism was detected as showed by arrowheads.

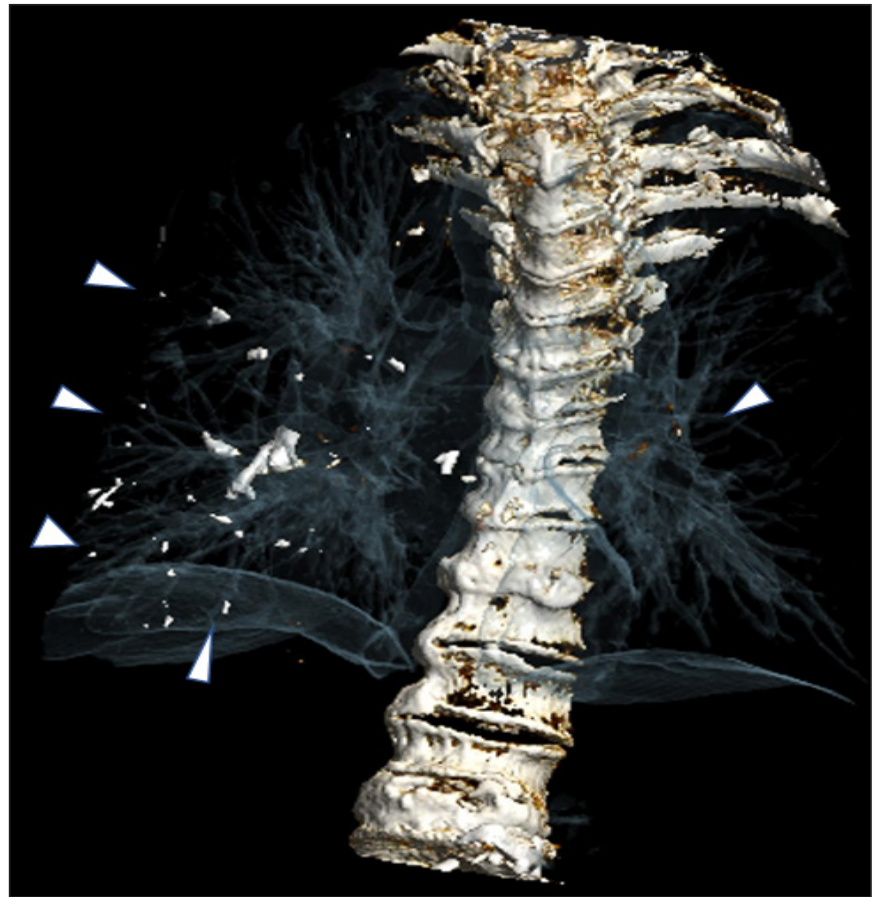

Figure 3. Multiple pulmonary cement embolisms were detected in the volume-rendered image compatible with vascular trace.
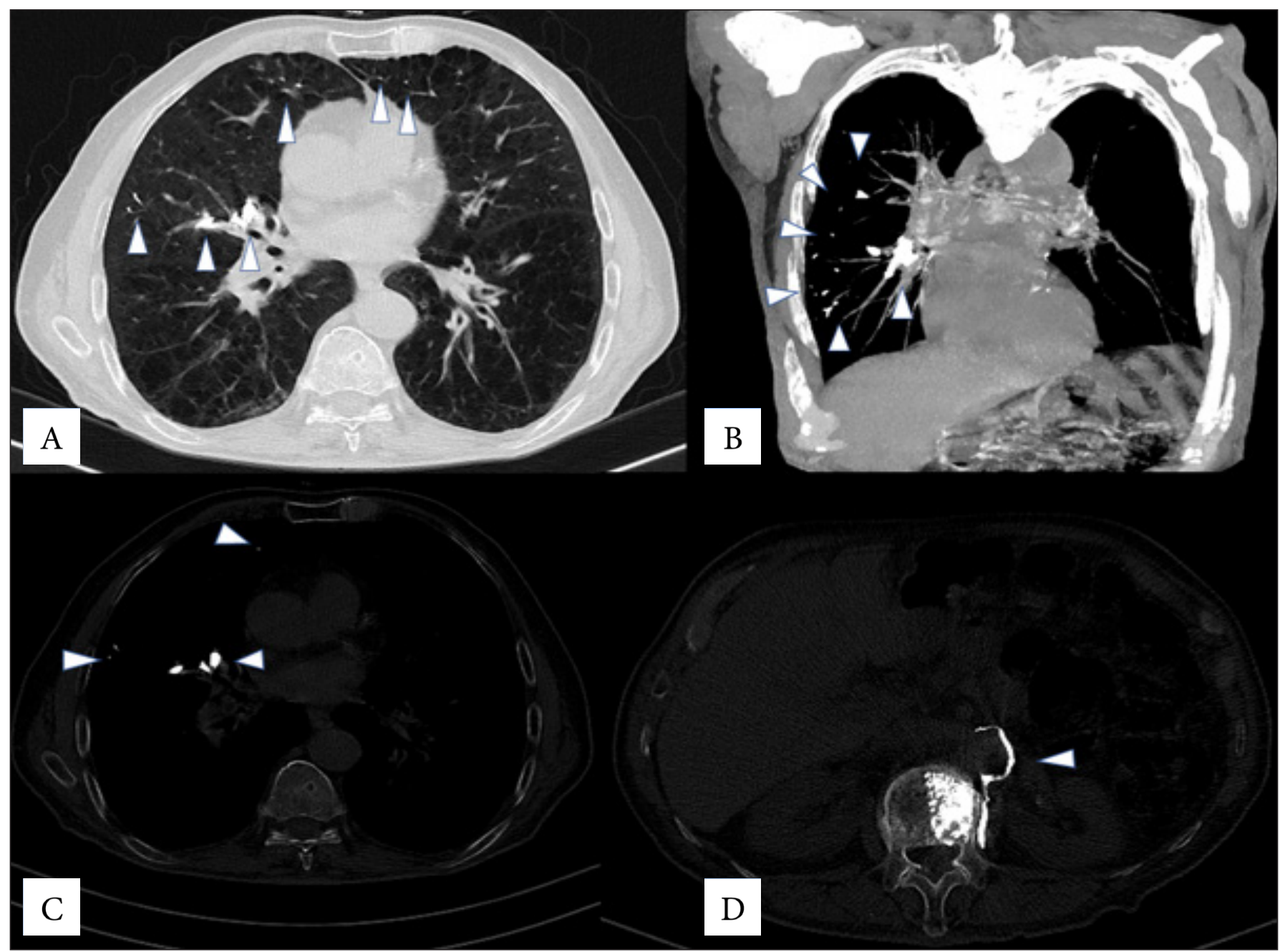

Figure 2. In the axial parenchymal window (a), coronal MIP (b), and axial bone window (c) images, multiple hyperdensities compatible with cement embolism were shown (arrowheads). The vertebroplasty place (L2 vertebra)and paravertebral venous cement embolism were demonstrated (d). 


\section{DISCUSSION}

Percutaneous vertebroplasty (PVP) is widely performed for the operative treatment of vertebral fractures in the last 30 years, which has gained popularity as a method for immediate pain relief (6). Transvertebral cement leakage into surrounding tissues and paravertebral veins are common complications after PVP. Linear hyperdensities of the cement embolism in segmental and subsegmental branches are seen on thoracic CT images in the bone window. PCE can be detected on plain film due to its excessive radiodensity and can be recognized by radiographic dense opacities in the lungs that have a tubular and branchial shape $(2,4,6)$.

Several studies and case reports are dealing with cement leakage and $\mathrm{CPE}$ in patients undergoing vertebroplasty. Yuan et al. (5) have reported massive left pulmonary embolism (PE) and thrombus in the inferior vena cava in a 65-year-old patient who had PVP of L2 vertebra due to osteoporosis. The patient complained of dyspnea and embolectomy was needed for treatment. Rahimi et al. (7) have reported a 38-year-old male patient accompanying Cushing's syndrome, who got treatment of PVP for his thoracic compression fractures due to osteoporosis. The patient was admitted to the hospital with sudden difficulty in breathing and chest pain. This patient was reported as osteoporotic and cement injection was approved to T7 and T12 vertebra corpus. Naud et al. (8) also reported a 55-year-old female patient who presented with chest pain and presyncope three weeks after a motor vehicle accident. Kyphoplasty was performed on the patient with L1 compression fracture. It was stated that the patient received anticoagulant medication for 6 months. Vertebral metastases are one of the other reasons for PVP. Mansour et al. (6) reported $12.7 \%$ PCE in their study of 102 cancer patients with malignant vertebral fractures and osteoporotic fractures and undergone PVP. They have reported PCE was more common with multiple myeloma. In the literature, there is also a case report presented with calcified mass in the right atrium due to PVP depended on the septal wall (9). Siddique et al. (10) reported PCE in the 57-year-old male patient who had bilateral lung transplantation secondary to idiopathic pulmonary fibrosis and had PVP due to chronic back pain secondary to chronic vertebral compression fractures.

Characteristic radiological findings are multiple tubular or branching radio-opacities in PCE. It can be easily detected on radiographs, due to its extremely radiodensity. Unenhanced CT scans are characterized by nodules or intraluminal tubular hyperdense materials (11). Since PMMA shows high attenuating on CT, PCE is better detected on CT images without contrast or portal venous phase. CT is also highly successful in demonstrating the extruded cement also in other venous system structures (12).

\section{CONCLUSION}

Pulmonary cement embolism (PCE) after vertebroplasty is a well-known complication that usually remains asymptomatic. PCE must be treated operatively or nonoperatively with anticoagulation if it is symptomatic. In symptomatic cases, it is important to be recognized by the radiologist so that timely treatment can be done. Although direct radiography is mostly sufficient, CT is the most useful technique, especially in terms of more accurate and detailed demonstration efflux of cement to the venous system and pulmonary circulation. It is obvious that if the radiologist is not informed about vertebroplasty, if it is evaluated only with thoracic $\mathrm{CT}$, diagnosis will be difficult. In addition, it should be kept in mind by the radiologist that the cement-related material cannot be distinguished from the contrast in the lumen at the soft tissue window due to its hyperdense feature, and it becomes visible especially at the bone window.

\section{ETHICAL DECLARATIONS}

Informed Consent: Written informed consent was obtained from all participants who participated in this study.

Referee Evaluation Process: Externally peer-reviewed.

Conflict of Interest Statement: The authors have no conflicts of interest to declare.

Financial Disclosure: The authors declared that this study has received no financial support.

Author Contributions: All of the authors declare that they have all participated in the design, execution, and analysis of the paper, and that they have approved the final version.

\section{REFERENCES}

1. Deramond H, Depriester H, Galibert P, Le Gars D. Percutaneous vertebroplasty with polymethylmethacrylate: Technique, indication, and results. Radiol Clin North Am 1998; 36: 533-46.

2. Habib N, Maniatis T, Ahmed S, et al. Cement pulmonary embolism after percutaneous vertebroplasty and kyphoplasty: an overview. Heart Lung 2012; 41: 509-11.

3. Unal E, Balci S, Atceken Z, Akpinar E, Ariyurek OM. Nonthrombotic pulmonary artery embolism: imaging findings and review of the literature. AJR Am J Roentgenol 2017; 208: 505-16.

4. Ignacio JMF, Ignacio KHD. Pulmonary embolism from cement augmentation of the vertebral body. Asian Spine J 2018; 12: 3807.

5. Yuan Z, Zhou Y, Zhou X, et al. Severe pulmonary embolism was secondary to cement inferior vena cava embolism after percutaneous vertebroplasty. Ann Vasc Surg 2018; 48: 255.e1-e3.

6. Mansour A, Abdel-Razeq N, Abuali H, et al. Cement pulmonary embolism as a complication of percutaneous vertebroplasty in cancer patients. Cancer Imaging 2018; 18: 5. 
7. Rahimi B, Boroofeh B, Dinparastisaleh R, Nazifi H. Cement pulmonary embolism after percutaneous vertebroplasty in a patient with cushing's syndrome: A case report. Respir Med Case Rep 2018; 25: 78-85.

8. Naud R, Guinde J, Astoul P.Pulmonary cement embolism complicating percutaneous kyphoplasty: a case report. Respir Med Case Rep 2020; 31: 101188.

9. Kim DH, Kim SS, Kim HK. Cement emboli presenting as right atrial mass caused by percutaneous vertebroplasty. Acta Cardiol Sin 2020; 36: 390-3.

10. Siddiqui A.S, Goodarzi A, Mamjumdar T, et al. A rare case of pulmonary cement embolism in a lung transplant patient. Respir Med Case Rep 2018; 24: 63-4.

11. Seo JS, Kim YJ, Choi BW, Kim TH, Choe KO. MDCT of pulmonary embolism after percutaneous vertebroplasty. Am J Roentgenol 2005; 184: 1364-5.

12. Gangi A, Guth S, Imbert JP et-al. Percutaneous vertebroplasty: indications, technique, and results. Radiographics 2003; 23: e10 